\title{
Relaxation and Nonoccurrence of the Lavrentiev Phenomenon for Nonconvex Problems
}

\author{
Farhad HÜSSEINOV \\ Department of Economics, Bilkent University, 06800 Ankara, Turkey \\ E-mail: farhad@bilkent.edu.tr
}

\begin{abstract}
The paper studies a relaxation of the basic multidimensional variational problem, when the class of admissible functions is endowed with the Lipschitz convergence introduced by Morrey. It is shown that in this setup, the integral of a variational problem must satisfy a classical growth condition, unlike the case of uniform convergence. The relaxations constructed here imply the existence of a Lipschitz convergent minimizing sequence. Based on this observation, the paper also shows that the Lavrentiev phenomenon does not occur for a class of nonconvex problems.
\end{abstract}

Keywords Multidimensional variational problem, relaxation, Lavrentiev phenomenon MR(2010) Subject Classification 49J45, 52A41

\section{Introduction}

Let $(X, \tau)$ be a topological space and consider a pair of real functions $I, J$ defined on $X$. The (abstract) variational problem $P_{2}: \inf \{J(x): x \in X\}$ is said to be a relaxation of $P_{1}: \inf \{I(x)$ : $x \in X\}$ if (i) $J(x) \leq I(x)$ for each $x \in X$; and (ii) for each $x \in X$, there exists a sequence $x_{k}$ in $X(k \in N)$ such that $J(x)=\lim _{k} I\left(x_{k}\right)$ and $x=\tau-\lim _{k} x_{k}$. A point $x_{0}$ in $X$ is said to be a generalized solution of the problem $P_{1}$ if there exists a minimizing sequence of $P_{1}$ that converges to $x_{0}$. Clearly, point $x_{0}$ is a generalized solution to problem $P_{1}$ if and only if it is a solution to problem $P_{2}$.

Given two topologies $\tau_{1}, \tau_{2}$ on $X$, if $\tau_{1}$ is weaker than $\tau_{2}$, a relaxation with respect to $\tau_{2}$ is also a relaxation with respect to $\tau_{1}$. Put differently, strengthening the topology on $X$ narrows the class of relaxations of a given problem. In particular, in the extreme case of the discrete topology, i.e., the strongest topology on $X$, there does not exist a variational problem that admits a relaxation which is distinct from itself. Thus, in this case, the notion of a generalized solution coincides with the classical solution concept. More generally, the stronger the topology on $X$, the closer the generalized solution to the classic one, in a certain sense. Therefore, when dealing with a variational problem that does not have a (classical) solution, it is natural to search for a generalized solution in the strongest possible topology.

On the other hand, narrowing down the set of admissible elements $X$ in a variational problem may be useful on occasion. Suppose, for instance, that $P_{20}: \inf \left\{J_{0}(x): x \in X_{0}\right\}$ is a relaxation of problem $P_{10}: \inf \left\{I(x): x \in X_{0}\right\}$ where $X_{0}$ is a subset of $X$ that represents the set of (regular) admissible elements. Let us also assume that the function $J_{0}$ admits an extension 
$J$ to $X$ such that (i) $J(x) \leq I(x)$ for every $x \in X$; and (ii) the problem $P_{2}: \inf \{J(x): x \in X\}$ has a solution that belongs to $X_{0}$ (regularity theory). These conditions would imply that there exists a regular minimizing sequence for problem $P_{1}$ that converges in the underlying topology $\tau$ to an element of $X_{0}$, namely to the solution of $P_{2}$. In particular, the $\left(X-X_{0}\right)$-Lavrentiev phenomenon could be ruled out in the original problem $P_{1}$. Theorems 4.1 and 4.2 below examplify such a combination of the relaxation and the regularity theories. A crucial step in the implemention of this scheme is to "translate" the assumptions on the data of problem $P_{1}$ to those of problem $P_{2}$ (see, for example, Lemma 4.2 below).

In this paper, we study a relaxation of the basic multidimensional variational problem, when the class of admissible functions is endowed with the Lipschitz convergence. It turns out that, unlike the case of uniform convergence, this setup requires the integrand to satisfy a superlinear growth condition that is familiar from the theory of existence of a solution. As we have argued above, in this topology the existence of a relaxation that has a Lipschitz solution implies the existence of a Lipschitz converging minimizing sequence for the original problem at hand. Such "regularity" of a minimizing sequence can be seen as a substitute for the notion of a regular solution, just as a minimizing convergent sequence is considered as a substitute for the classical solution concept. Two features of the notion of a regular minimizing sequence are worth mentioning: (i) each function in the sequence possesses some nice properties of a regular solution in the traditional sense such as being Lipschitz or of class $C^{1}$; (ii) the sequence converges in some strong topology.

Most importantly, we show here that the existence of a regular minimizing sequence rules out the Lavrentiev phenomenon (LP) for some classes of nonconvex integrands. In 1926, Lavrentiev [1] constructed an example of a one-dimensional integral functional $J(x)$ having the following property:

$$
\inf \{J(x): x \in A C\}<\inf \left\{J(x): x \in C^{2}\right\},
$$

where $A C$ and $C^{2}$ denote, respectively, the set of absolutely continuous and twice continuously differentiable functions on an interval $[a, b]$ that satisfy some boundary conditions. It is easy to show that the latter infimum coincides with the one over the class of Lipschitz functions. A somewhat simpler example exhibiting the LP was given by Mania in 1934 (see [2, p. 514]). Tihomirov [3] offered a series of even simpler examples of LP. In the examples of Mania and Tihomirov the integrands are convex in the velocity variable, but they are not coercive. Ball and Mizel [4] suggested an example of LP where the integrand is strictly convex and coercive, the requirements of a classical existence theorem due to Tonelli. Loewen [5] devised a simple trick for deriving such an integrand from Mania's example. Applying this trick to Tihomirov's example, one can obtain an even simpler instance of LP that satisfies the assumptions of the existence theorem. Buttazzo and Mizel [6] suggested treating the Lavrentiev gap as a relaxation phenomenon. For developments concerning the LP, see the surveys by Buttazzo and Belloni [7] and Mizel [8], and papers [9-13].

The importance of LP from the viewpoint of numerical calculation is discussed by Ball and Knowles [14], Loewen [5] and Clarke [15]. Indeed, when applied to problems displaying LP, the usual numerical methods often fail because they use suitable classes of Lipschitz functions for approximation. Ball and Knowles [14] offered a special method for handling this problem. 
Similar difficulties arise when one tries to calculate numerically a non-regular convergent minimizing sequence. An essential factor for the success of this sort of a calculation is the uniform boundedness of derivatives which follows from the Lipschitz convergence criterion demanded by the definition of a regular minimizing sequence that we study in this paper.

Proofs of the main results are based on some preliminary findings on spannability of functions which we present in the next section. In the third section, we apply these findings to relaxation of multidimensional variational problems in which the set of admissible functions is endowed with Lipschitz convergence. In the last section, we establish the existence of a regular minimizing sequence for one-dimensional variational problems and relate this finding to non-occurrence of Lavrentiev phenomenon.

\section{Spannability}

Set $\overline{\mathbb{R}}=\mathbb{R} \cup\{+\infty\}$, and let epi $f$ denote the epigraph of a function $f: \mathbb{R}^{n} \rightarrow \overline{\mathbb{R}}$. The convex envelope conv $f$ and the convexification $f^{* *}$ of $f$ are defined as the greatest convex function and the greatest lower semicontinuous convex function not exceeding $f$ on $\mathbb{R}^{n}$, respectively. Notation $f^{* *}$ refers to the fact that the convexification of $f$ coincides with its second conjugate by Young-Fenchel (see [16]).

Recall that a function $f: \mathbb{R}^{n} \rightarrow \overline{\mathbb{R}}$ is said to be spannable if for each point $x \in \mathbb{R}^{n}$ there exist points $x_{1}, \ldots, x_{n} \in \mathbb{R}^{n}$ and nonnegative numbers $\lambda_{1}, \ldots, \lambda_{n}$ with $\lambda_{1}+\cdots+\lambda_{n}=1$ such that

$$
x=\sum_{i=1}^{m} \lambda_{i} x_{i} \quad \text { and } \quad f^{* *}(x)=\sum_{i=1}^{m} \lambda_{i} f\left(x_{i}\right) .
$$

This is equivalent to saying that the graph of $f^{* *}$ is contained in the convex hull of the graph of $f$.

Following Klee [17], we say that a set $A$ in $\mathbb{R}^{n}$ is coterminal with a ray $\rho=\{x+r y: r>0\}$ provided $\sup \{r: r>0$ and $x+r y \in A\}=\infty$.

The following theorem on the spannability is proved in Husseinov [18]:

Theorem 2.1 Let $f: \mathbb{R}^{n} \rightarrow \overline{\mathbb{R}}$ be a lower semi-continuous function such that epi $f^{* *}$ contains no line. Then $f$ is spannable if, and only if, its graph is coterminal with every nonvertical extreme ray of the epigraph of $f^{* *}$.

It is easy to show that superlinear coercivity is preserved under convexification. On the other hand, the graph of a superlinearly coercive convex function can not contain a nonvertical extreme ray. Therefore, by Theorem 2.1, a superlinearly coercive lower semicontinuous function is spannable, which is a well-known result (see [16-21]).

We generalize Theorem 2.1 for a function depending on a parameter varying in a topological space. This generalization simultaneously specifies the representation of convexification $f^{* *}$ given in Theorem 2.1; it shows that for any bounded part $K$ of $\mathbb{R}^{n}$, there is a ball $B$ such that for the points from $K$ the representation in question involves only points of this ball $B$. We would like to stress here that the central result of the present paper on relaxation is intimately related to these results on spannability.

Cellina [21] proves a spannability theorem (Theorem 1 in [21]) which is a straightforward consequence of Theorem 2.1. Theorem 1 in [21] assumes the following Bounded Intersection 
Property (BIP): If for every $\bar{x} \in \mathbb{R}^{n}$, there exists $\bar{p} \in \partial f^{* *}(\bar{x})$ such that

$$
\left\{x \in \mathbb{R}^{n}: f^{* *}(x)=f^{* *}(\bar{x})+\bar{p} \cdot(x-\bar{x})\right\}
$$

is bounded. Here $\partial f^{* *}(\bar{x})$ denotes the subdifferential of function $f^{* *}$ at point $\bar{x}$. It is easy to see that BIP implies that the epigraph epi $f^{* *}$ contains no extreme rays. Indeed, assuming that $r=\{a+\beta b: b \beta \geq 0\}$ is an extreme ray of epi $f^{* *}$, we will have

$$
r \subset\left\{x \in R^{n}: f^{* *}(x)=f^{* *}(\bar{x})+\bar{p} \cdot(x-\bar{x})\right\}
$$

for every point $\bar{x}$ in the relative interior of $r$ and $\bar{p} \in \partial f^{* *}(\bar{x})$, which contradicts BIP. Note also that the assumption of continuity of function $f$ in Theorem 1 of [21] can be relaxed to the lower semicontinuity.

Corollary 2.2 ([21, Theorem 1]) Let $f$ be a continuous function, affinely minorized, satisfying BIP. Then, for every $\bar{x} \in \mathbb{R}^{n}$, there exist at most $m \leq n+1$ points $x_{i}$ and coefficients of a convex combination $\lambda_{i}$ such that

$$
\bar{x}=\sum_{i=1}^{m} \lambda_{i} x_{i} \quad \text { and } \quad f^{* *}(\bar{x})=\sum_{i=1}^{m} \lambda_{i} f\left(x_{i}\right) .
$$

Moreover, $x_{i}$ belongs to $\left\{x \in \mathbb{R}^{n}: f(x)=f^{* *}(\bar{x})+\bar{p} \cdot(x-\bar{x})\right\}$.

Corollary 2.3 Let $f: \mathbb{R}^{n} \rightarrow \mathbb{R}$ be a lower semicontinuous function satisfying the following superlinear growth condition

$$
\lim _{\|x\| \rightarrow \infty} f(x) /\|x\|=\infty .
$$

Then for an arbitrary point $x \in \mathbb{R}^{n}$,

$$
f^{\star \star}(x)=\min \left\{\sum_{i=1}^{n+1} \lambda_{i} f\left(x_{i}\right): \sum_{i=1}^{n+1} \lambda_{i} x_{i}=x,\left(\lambda_{1}, \ldots, \lambda_{n+1}\right) \in S_{n}\right\} .
$$

We will need one auxiliary result on the estimation of the subdifferential of a convex function.

Lemma 2.4 Let $B$ be a convex closed body in $\mathbb{R}^{n}, f: B \rightarrow \mathbb{R}$ be a convex function and $B_{0}$ be a subset of $B$, such that $B_{0}$ is compact and $B_{0} \subset$ int $B$. Then

$$
\sup \left\{\left\|y^{\star}\right\|: y^{\star} \in \partial f(y), y \in B_{0}\right\} \leq \frac{M}{d},
$$

where $M=\sup \left\{\left|f\left(y_{1}\right)-f\left(y_{2}\right)\right|: y_{1} \in \partial B_{1}, y_{2} \in \partial B_{0}\right\}$ and $d=\operatorname{dist}\left(B_{0}, \partial B\right)$.

Proof Denote by $S^{1}$ the unit sphere in $\mathbb{R}^{n}$. Fix $x \in B_{0}$ and let $h \in S^{1}$ be an arbitrary vector. Let $l$ be the straight line passing through $x$ and parallel to vector $h$. Then the convexity of function $f$ prompts

$$
\left|f^{\prime}(y, h)\right| \leq \max \left\{\frac{\mid f(d)-f(b)}{|d-b|}, \frac{|f(a)-f(c)|}{|a-c|}\right\} \leq \frac{M}{d} .
$$

Thus

$$
\left|f^{\prime}(y, h)\right| \leq \frac{M}{d}, \quad \forall h \in S^{1} .
$$

Then by the well-known fact (see [22, Theorem 23.4]),

$$
\sup _{y^{\star} \in \partial f(y)}\left\langle y^{\star}, h\right\rangle=f^{\prime}(y, h) \leq \frac{M}{d}, \quad \forall h \in S^{1}
$$


or

$$
\left\langle y^{\star}, h\right\rangle \leq \frac{M}{d}, \quad \forall h \in S^{1}, \forall y^{\star} \in \partial f(y) .
$$

From this relation, it easily follows

$$
\left\|y^{\star}\right\| \leq \frac{M}{d}, \quad \forall y^{\star} \in \partial f(y) .
$$

We now formulate a uniform variant of Theorem 2.1 and prove it for a function depending on a parameter. Simultaneously, this result specifies Theorem 2.1.

Theorem 2.5 Let $T$ be a topological space and $f: T \times \mathbb{R}^{n} \rightarrow \mathbb{R}$. Let function $f(\tau, \cdot): \mathbb{R}^{n} \rightarrow \mathbb{R}$ be bounded from below on any compact from $\mathbb{R}^{n}$ and satisfy the growth condition (2.1) locally uniformly in $\tau \in T$. Moreover, let functions $f(\tau, \cdot)$ be lower semicontinuous for all $\tau \in T$. Then, if $T_{0} \subset T$ is a compact subset, for an arbitrary $r>0$, there exists $R>0$ such that

a) For each $\tau \in T_{0}$, and $y \in B_{r}(0)$, there exist $y_{1}, \ldots, y_{n+1} \in B_{R}(0)$ and $\left(\lambda_{1}, \ldots, \lambda_{n+1}\right) \in S_{n}$ such that

$$
y=\sum_{i=1}^{n+1} \lambda_{i} y_{i} \quad \text { and } \quad f^{\star \star}(\tau, y)=\sum_{i=1}^{n+1} \lambda_{i} f\left(\tau, y_{i}\right) .
$$

In other words,

$$
\left.f^{\star \star}(\tau, \cdot)\right|_{B_{r}(0)}=\left.f_{R}^{\star \star}(\tau, \cdot)\right|_{B_{r}(0)} \quad \text { for } \tau \in T_{0},
$$

and the representation above holds with

$$
f_{R}(\tau, y)= \begin{cases}f(\tau, y), & \text { for }\|y\| \leq R \\ \infty, & \text { for }\|y\|>R\end{cases}
$$

b) If at least one of the points $y_{1}, \ldots, y_{n+1}$ does not belong to $B_{R}(0)$ and the corresponding coordinate of vector $\left(\lambda_{1}, \ldots, \lambda_{n+1}\right) \in S_{n}$ is positive, then for $y=\sum_{i=1}^{n+1} \lambda_{i} y_{i}$,

$$
f^{\star \star}(\tau, y)<\sum_{i=1}^{n+1} \lambda_{i} f\left(\tau, y_{i}\right) \quad \text { for } \tau \in T_{0} .
$$

Proof Fix an arbitrary point $\tau_{0} \in T_{0}$. By the above assumption, there exists a neighbourhood $U\left(\tau_{0}\right)$ of $\tau_{0}$ such that functions $f(\tau, \cdot)\left(\tau \in U\left(\tau_{0}\right)\right)$ are bounded on any compact $K$ in $\mathbb{R}^{n}$ and satisfy the growth condition (2.1) uniformly in $\tau \in U\left(\tau_{0}\right)$. Denote

$$
M=2 \sup \left\{|f(\tau, y)|: \tau \in U\left(\tau_{0}\right), y \in B_{r+1}(0)\right\} .
$$

For any points $\tau \in U\left(\tau_{0}\right)$ and $y \in B_{r}(0)$, fix some support hyperplane $\Pi_{y}(\tau)$ to epi $f^{\star \star}(\tau, \cdot)$ at the point $\left(y, f^{\star \star}(\tau, y)\right)$. Obviously, it is not vertical and therefore is the graph of some affine function $l_{(\tau, y)}\left(y^{\prime}\right)=\left(a_{y}(\tau), y^{\prime}\right)+b y(\tau)$. By Lemma 2.4,

$$
a=\sup \left\{\left\|a_{y}(\tau)\right\|: \tau \in U\left(\tau_{0}\right), y \in B_{r}(0)\right\}<M<\infty .
$$

Consequently,

$$
b=\sup \left\{b_{y}(\tau): \tau \in U\left(\tau_{0}\right), y \in B_{r}(0)\right\}<(r+1) M<\infty .
$$

It follows from the growth condition that there exists a number $R_{0}>0$ such that $f\left(\tau, y^{\prime}\right)>$ $a\left\|y^{\prime}\right\|+b$ for $\tau \in U\left(\tau_{0}\right),\left\|y^{\prime}\right\| \geq R_{0}$, and therefore,

$$
f\left(\tau, y^{\prime}\right)>l_{\left(\tau, y^{\prime}\right)}\left(y^{\prime}\right) \quad \text { for } \tau \in U\left(\tau_{0}\right), y^{\prime} \in B_{R_{0}}(0), y \in B_{r}(0) .
$$


Thus, for any point $\tau \in T_{0}$, there exist its neighbourhood $U(\tau)$ and a number $R(\tau)$ such that (2.2) is satisfied with the replacement $U\left(\tau_{0}\right)$ by $U(\tau)$ and $R_{0}$ by $R(\tau)$, respectively. Choose a finite subcover $\left\{U\left(\tau_{1}\right), \ldots, U\left(\tau_{n}\right)\right\}$ of the cover $\left\{U(\tau): \tau \in T_{0}\right\}$ of compact $T_{0}$ and denote $R=\max \left\{R\left(\tau_{k}\right): k=1, \ldots, N\right\}$. Then

$$
f\left(\tau, y^{\prime}\right)>l_{(\tau, y)}\left(y^{\prime}\right) \quad \text { for } \tau \in T_{0}, y^{\prime} \in B_{R}(0), y \in B_{r}(0) .
$$

Fix an arbitrary point $\left(\tau_{0}, y_{0}\right) \in T_{0} \times B_{r}(0)$. By Theorem 2.1 , there exist points $y_{i} \in \mathbb{R}^{n}(i=$ $1, \ldots, n+1)$ and $\bar{\lambda}=\left(\lambda_{1}, \ldots, \lambda_{n+1}\right) \in S_{n}$ such that

$$
y_{0}=\sum_{i=1}^{n+1} \lambda_{i} y_{i} \quad \text { and } \quad f^{\star \star}\left(\tau_{0}, y_{0}\right)=\sum_{i=1}^{n+1} \lambda_{i} f\left(\tau_{0}, y_{i}\right) \text {. }
$$

We assert that $y_{i} \in B_{R}(0)(i=1, \ldots, n+1)$. Suppose on the contrary, there exists index $i_{0}$ such that $y_{i_{0}} \notin B_{R}(0)$. Then from $(2.3)$, we get

$$
f\left(\tau_{0}, y_{i_{0}}\right)>l_{\left(\tau_{0}, y\right)}\left(y_{i_{0}}\right) \quad \text { for } y \in B_{r}(0) .
$$

In particular, for $y=y_{0}$, we get that $\left(y_{i_{0}}, f\left(\tau_{0}, y_{i_{0}}\right)\right)$ is contained in upper open halfspace $\Pi_{y_{0}}^{+}\left(\tau_{0}\right)$, defined by hyperplane $\Pi_{y_{0}}\left(\tau_{0}\right)$. But it is obvious that for remaining indices $i$, point $\left(y_{i}, f\left(y_{i}\right)\right)$ belongs to the closed upper half-space $\bar{\Pi}_{y_{0}}^{+}\left(\tau_{0}\right)$. It is easy to conclude from this that point $\left(y_{0}, f^{\star \star}\left(\tau_{0}, y_{0}\right)\right)=\left(\sum_{i=1}^{n+1} \lambda_{i} y_{i}, \sum_{i=1}^{n+1} \lambda_{i} f\left(\tau_{0}, y_{i}\right)\right)$ belongs to the open half-space $\Pi_{y_{0}}^{+}\left(\tau_{0}\right)$. But this contradicts the inclusion $\left(y_{0}, f^{\star \star}\left(\tau_{0}, y_{0}\right)\right) \in \Pi_{y_{0}}\left(\tau_{0}\right)$.

b) Suppose the opposite: for any $R_{1}>0$, there exist $y_{i}(i=1, \ldots, n+1)$ and vector $\left(\lambda_{1}, \ldots, \lambda_{n+1}\right) \in S_{n}$, such that $y_{0}=\sum_{i=1}^{n+1} \lambda_{i} y_{i}$ and $f^{\star \star}(\tau, y) \leq \sum_{i=1}^{n+1} \lambda_{i} f\left(\bar{\tau}, y_{i}\right), \exists \bar{\tau} \in T_{0}$, where $y_{i_{0}} \in B_{r_{1}}(0)$ for some $i_{0} \in\{1, \ldots, n+1\}$. Putting $R_{1}=R$, where number $R$ is chosen as in the proof of point a), $\tau_{0}=\bar{\tau}$, and repeating reasoning of the proof of point a) beginning from the formula (2.4), we prove b).

In the case, when the space $T$ consists of a single point, Theorem 2.5 transforms to the following

Corollary 2.6 Let a function $f: \mathbb{R}^{n} \rightarrow \mathbb{R}$ satisfy the growth condition (2.1) and be bounded on any compact subset of $\mathbb{R}^{n}$. Then for any number $r>0$ there exists $R>0$ such that, for any point $y \in B_{r}(0)$ there exist vectors $y_{1}, \ldots, y_{n+1} \in B_{R}(0)$ and $\left(\lambda_{1}, \ldots, \lambda_{n+1}\right) \in S_{n}$ such that

$$
y=\sum_{i=1}^{n+1} \lambda_{i} y_{i} \quad \text { and } \quad f^{\star \star}(y)=\sum_{i=1}^{n+1} \lambda_{i} f\left(y_{i}\right) .
$$

If Theorem 2.1 asserts that to represent a value of convexification $f^{\star \star}$ at a point $y$ it is sufficient to restrict within some bounded part of the space $\mathbb{R}^{n}$, Corollary 2.6 shows that this is true for any bounded part of the space $\mathbb{R}^{n}$ instead of for a single point. In other words, Theorem 2.1 asserts that for any $y \in \mathbb{R}^{n}$ there exists $R>0$ such that, $f^{\star \star}(y)=\left(\left.f\right|_{B_{R}}(0)\right)^{\star \star}(y)$, whereas Corollary 2.6 asserts that, for any $r>0$, there exists $R>0$ such that

$$
f^{\star \star}(y)=\left(\left.f\right|_{B_{R}}(0)\right)^{\star \star}(y) \text { for all } y \in B_{r}(0) .
$$

Remark 2.7 It is obvious that one can count that a function $R(\cdot)$ is monotonically increasing. 


\section{Relaxation}

Now we consider the basic multidimensional variational problem in a slightly generalized form. First, we introduce some notations. Let $\Omega$ be an arbitrary bounded open subset of $\mathbb{R}^{n}, \bar{\Omega}$ and $\partial \Omega$ its closure and boundary, respectively, and let $\Gamma$ be an arbitrary subset of the boundary $\partial \Omega$. Denote by $W_{\infty}^{1}(\Omega)$ the space of Lipschitz functions defined on $\bar{\Omega}$. Denote also by $L_{\infty}^{n}(\Omega)$ and $L_{1}^{n}(\Omega)$ the linear normed spaces of essentially bounded and integrable $n$-vector-functions defined on $\Omega$. Norm of space $L_{\infty}^{n}(\Omega)$ will be denoted by $\|\cdot\|_{\infty}$. In the case $n=1$, the upper index in all three notations will be omitted.

Let $f: \bar{\Omega} \times \mathbb{R} \times \mathbb{R}^{n}$ be continuous and let $\varphi: \Gamma \rightarrow \mathbb{R}$ be arbitrary fixed functions. Consider the following multidimensional basic variational problem

$$
\left\{\begin{array}{l}
J_{f}(x(\cdot))=\int_{\Omega} f\left(t, x(t), x^{\prime}(t) d t \rightarrow \mathrm{inf}\right. \\
\left.x(\cdot)\right|_{\Gamma}=\varphi
\end{array}\right.
$$

where $x^{\prime}(t)$ denotes the gradient of Lipschitz function $x(\cdot)$ defined on domain $\bar{\Omega}$, at the point $t \in \Omega$. In this section, problem (P) will be considered with the set of admissible functions $x(\cdot) \in W_{\infty}^{1}(\Omega)$.

The main purpose of this point is to construct a relaxation of problem $(\mathrm{P})$ when the set of admissible functions in this problem $E(\varphi)=\left\{x(\cdot) \in W_{\infty}^{1}(\Omega):\left.x(\cdot)\right|_{\Gamma}=\varphi\right\}$ is considered with the topology of the Lipschitz convergence. It turns out that, in this case, integrand $f$ should satisfy some growth condition on the third variable, traditional in the theory of existence of solutions of variational problems. Example 3.7 below shows that this condition is minimal.

We shall suppose that integrand $f$ is continuous and satisfies the growth condition

$$
\lim _{\|\zeta\| \rightarrow \infty} f(t, x, \zeta) /\|\zeta\|=\infty
$$

locally uniformly in $(t, x)$.

Definition 3.1 (Morrey [23, p. 113]) A sequence $x_{n}(\cdot), n=1,2, \ldots$, in $W_{\infty}^{1}(\Omega)$ is said to converge Lipschitz to $x(\cdot) \in W_{\infty}^{1}(\Omega)$, if $x_{n}(\cdot)$ converges uniformly to $x(\cdot)$ and $x_{n}(\cdot), n=1,2, \ldots$, satisfy the uniform Lipschitz condition.

Remark 3.2 In fact, the Lipschitz convergence is a convergence in a $\tau$-topology defined in the following way: the base of neighborhoods of $0 \in W_{\infty}^{1}(\Omega)$ is a family of subsets $T=\left\{U_{\varepsilon, V}\right\}$, where $U_{\varepsilon, V}=\left\{x(\cdot) \in W_{\infty}^{1}(\Omega):\|x(\cdot)\|_{\infty}<\varepsilon, x^{\prime}(\cdot) \in V\right\}$ for a positive number $\varepsilon$ and open neighbourhood $V$ of $0 \in L_{\infty}^{n}(\Omega)$ in $\star$-weak topology. It is clear that the Lipschitz convergence implies $*$-weak convergence of derivatives and then a convergence in topology $\tau$. Conversely, since $\star$-weak convergence of a sequence in $L_{\infty}^{n}(\Omega)$ implies its boundedness, we have that $\tau$ convergence implies the Lipschitz convergence. On the other hand, the Lipschitz convergence is a convergence in $\star-W_{\infty}^{1}$ topology (in the notations of Marcellini-Sbordone [24], $\star-H^{1-\infty}$ topology). Apparently, $\tau$-convergence implies $\star-W_{\infty}^{1}$ convergence. Conversely, it is a trivial consequence of Arzela's theorem that $\star-W_{\infty}^{1}$ convergence implies $\tau$-convergence.

Since the restriction of $\star$-weak topology to any part of space $L_{\infty}^{n}(\Omega)$ is metrizable (see [25, p. 426]), for an arbitrary positive number $M$, the subset $\left\{x(\cdot) \in W_{\infty}^{1}(\Omega):\left\|x^{\prime}(\cdot)\right\|_{\infty} \leq M\right\}$ of $W_{\infty}^{1}(\Omega)$ is metrizable. 
Furthermore, we shall assume that the set of admissible functions $E(\varphi)$ is endowed with the Lipschitz convergence.

Along with problem $(\mathrm{P})$, we consider the following problem

$$
\left\{\begin{array}{l}
J_{f}^{\star \star}(x(\cdot))=\int_{\Omega} f^{\star \star}\left(t, x(t), x^{\prime}(t)\right) d t \rightarrow \text { inf; } \\
\left.x(\cdot)\right|_{\Gamma}=\varphi
\end{array}\right.
$$

where $f^{\star \star}$ is a convexification of the integrand with respect to the third variable $\zeta \in \mathbb{R}^{n}$.

As in problem $(\mathrm{P})$, the set of admissible functions in $\left(\mathrm{PR}_{1}\right)$ is $E(\varphi)$.

Theorem 3.3 Let integrand $f: \bar{\Omega} \times \mathbb{R} \times \mathbb{R}^{n} \rightarrow \mathbb{R}$ satisfy the growth condition (3.1) and let the set of admissible in problem $(\mathrm{P})$ functions $E(\varphi)$ be endowed with the topology of the Lipschitz convergence. Then problem $\left(\mathrm{PR}_{1}\right)$ is a lower semicontinuous relaxation of problem $(\mathrm{P})$.

For the proof of Theorem 3.3, we need the following:

Lemma 3.4 Let $T$ be a locally compact metric space, and let $f: T \times \mathbb{R}^{n} \rightarrow \mathbb{R}$ be continuous and satisfy the growth condition

$$
\lim _{\|\zeta\| \rightarrow \infty}\|f(t, \zeta)\| /\|\zeta\|=\infty
$$

locally uniformly with respect to $t \in T$. Then function $f^{\star \star}$ is continuous and

$$
\lim _{\|\zeta\| \rightarrow \infty}\left\|f^{\star \star}(t, \zeta)\right\| /\|\zeta\|=\infty
$$

locally uniformly with respect to $t \in T$.

Proof The proof of the continuity of $f^{\star \star}$ is based on part (a) of Theorem 2.5 and is omitted here. Fix $t_{0} \in T$ and $c>0$. By the assumption, there is $R>0$, such that

$$
\frac{f(t, y)}{\|y\|} \geq c \text { for all } B_{R}(0), t \in B_{R}\left(t_{0}\right) \text {. }
$$

It follows that there is a number $\alpha$ such that

$$
f(t, y) \geq c\|y\|-\alpha \text { for all } y \in \mathbb{R}^{n}, t \in B_{R}\left(t_{0}\right) .
$$

Thus, the convex function $c\|y\|-\alpha$ does not exceed function $f(t, \cdot)$. By the definition of the second conjugate $f^{\star \star}$, we have

$$
f^{\star \star}(t, y) \geq c\|y\|-\alpha \quad \text { for all } y \in \mathbb{R}^{n}, t \in B_{R}\left(t_{0}\right) .
$$

Therefore,

$$
\underline{\lim }_{\|y\| \rightarrow \infty} \inf _{t \in B_{R}\left(t_{0}\right)} \frac{f^{\star \star}(t, y)}{\|y\|} \geq c .
$$

Since $c>0$ is arbitrary this implies property (3.3) locally uniformly with respect to $t \in T$.

Remark 3.5 It is seen from the above proof that if function $f$ is bounded below and satisfies the growth condition (3.2) uniformly with respect to $t \in T$, then function $f^{\star \star}$ also will possess the same properties.

Proof of Theorem $3.3 \quad$ Fix $x(\cdot) \in E(\varphi)$, and denote $p=1+\|x(\cdot)\|_{C}$ and $r=1+\left\|x^{\prime}(\cdot)\right\|_{\infty}$. For a positive number $\rho$, put

$$
f_{\rho}(t, x, \zeta)= \begin{cases}f(t, x, \zeta), & \text { for }\|\zeta\| \leq \rho \\ \infty, & \text { for }\|\zeta\|>\rho\end{cases}
$$


By Theorem 2.5, there exists a number $R>r$ such that

$$
f^{\star \star}(t, x, \zeta)=f_{R}^{\star \star}(t, x, \zeta) \quad \text { for } t \in \Omega,|x|<p, \zeta \in B_{r}(0) .
$$

Consider problems $\left(\mathrm{P}^{\prime}\right)$ and $\left(\mathrm{PR}_{1}^{\prime}\right)$ obtained by adding the constraint

$$
x^{\prime}(t) \in B_{R}(0) \text { for a.a. } t \in \Omega,
$$

to problems $(\mathrm{P})$ and $\left(\mathrm{PR}_{1}\right)$, respectively.

Thus, in problems $\left(\mathrm{P}^{\prime}\right)$ and $\left(\mathrm{PR}_{1}^{\prime}\right)$, the set of admissible functions is

$$
E(R, \varphi)=\left\{x(\cdot) \in W_{\infty}^{1}(\Omega): x^{\prime}(t) \varphi\right\} .
$$

By Theorem 2 from [26], there exists a sequence of infinitely differentiable functions $x_{k}(\cdot) \in$ $E(R, \varphi)(k \in \mathbb{N})$ converging uniformly to $x(\cdot)$, and such that

$$
\lim _{k \rightarrow \infty} J_{f}\left(x_{k}(\cdot)\right)=\lim _{k \rightarrow \infty} J_{f_{R}}\left(x_{k}(\cdot)\right)=\int_{\Omega} f^{\star \star}\left(t, x(t), x^{\prime}(t)\right) d t .
$$

Taking into account the definitions of numbers $p, r$ and relation (3.3), we get

$$
\int_{\Omega} f_{R}^{\star \star}\left(t_{1}, x(t), x^{\prime}(t)\right) d t=\int_{\Omega} f^{\star \star}\left(t, x(t), x^{\prime}(t)\right) d t .
$$

The latter relation, together with (3.5), yields

$$
\lim _{k \rightarrow \infty} J_{f}\left(x_{k}(\cdot)\right)=J_{f^{\star \star}}(x(\cdot)) .
$$

Thus, for an arbitrary function $x(\cdot) \in E(\varphi)$, there exists a sequence of functions $x_{k}(\cdot) \in$ $E(\varphi)(k \in \mathbb{N})$ converging uniformly to $x(\cdot)$, and such that their gradients are uniformly bounded and (3.6) is satisfied. The theorem will be proved if we show that functional $J_{f^{\star \star}}(\cdot)$ is lower semicontinuous.

Let $p$ be an arbitrary positive number. Put

$$
\tilde{f}^{p}(t, x, \zeta)= \begin{cases}f^{\star \star}(t, x, \zeta), & \text { for }|x| \leq p, \\ f^{\star \star}(t, p \cdot \operatorname{sgn}(x-p), \zeta), & \text { for }|x|>p .\end{cases}
$$

Then it follows from the uniform variant of Lemma 3.4 (see Remark 3.5 after Lemma 3.4) that

$$
\lim _{\|\zeta\| \rightarrow \infty} \inf _{(t, x) \in \Omega \times \mathbb{R}} \tilde{f}_{p}(t, x, \zeta) /\|\zeta\|=\infty .
$$

By Lemma 3.4 integrand $f^{\star \star}$ is continuous jointly on variables, and therefore, integrand $\tilde{f}_{p}$ is also continuous. Thus, integrand $\tilde{f}_{p}$ satisfies all assumptions of the theorem on lower semicontinuity from Ekeland-Temam [16, p. 243].

It is easy to see that, if a sequence $x_{k}(\cdot) \in W_{\infty}^{1}(\Omega)(k \in \mathbb{N})$ converges Lipschitz to $x(\cdot)$, then the sequence of gradients of these functions $x_{k}^{\prime}(\cdot) \in L_{\infty}^{n}(\Omega)(k \in \mathbb{N})$ converges in $\sigma\left(L_{1}^{n}, L_{\infty}^{n}\right)$ topology to the vector-function $x^{\prime}(\cdot)$. It follows from that and the Lower Semicontinuity Theorem that functional $J_{\tilde{f}_{p}}(x(\cdot))=\int_{\Omega} \tilde{f}_{p}\left(t, x(t), x^{\prime}(t)\right) d t$ is lower semicontinuous relative to the Lipschitz convergence in space $W_{\infty}^{1}(\Omega)$. Note that functional $J_{f \star \star}(\cdot)$ coincides with functional $J_{\tilde{f}_{p}}(\cdot)$ on the ball $B_{p}(0) \subset W_{\infty}^{1}(\Omega)$. Since number $p$ was arbitrary, we get that functional $J_{f^{\star \star}}(\cdot)$ is lower semicontinuous on the entire space $W_{\infty}^{l}(\Omega)$. 
Corollary 3.6 Let problem $\left(\mathrm{PR}_{1}\right)$ have a solution. Then problem $(\mathrm{P})$ has a minimizing sequence Lipschitz converging to the solution of problem $\left(\mathrm{PR}_{1}\right)$.

Example 3.7 It is easy to see that for problem $(\mathrm{P})$ with $\Omega=[0,1], \varphi(0)=0, \varphi(1)=1, f(\zeta)=$ $2|\zeta|$ for $|\zeta| \leq 1$ and $f(\zeta)=2+|\zeta-\operatorname{sgn} \zeta|$ for $|\zeta|>1$, the assertion of Theorem 3.3 is not valid.

Remark 3.8 It follows from Serrin's theorem on lower semicontinuity (see [27]) that functional $J_{f^{\star \star}}(\cdot)$ of problem $\left(\mathrm{PR}_{1}\right)$ is lower semicontinuous relative to the topology of uniform convergence on $E(\varphi)$.

Remark 3.9 It is a mere technicality to generalize Theorem 3.1 to the case of normal integrands assuming value $+\infty$.

Marcellini-Sbordone [24, Theorem 4.4] obtained a result concerning a relaxation of problem (P) with the additional restriction $\left\|x^{\prime}(.)\right\|_{L_{\infty}} \leq r$. Since uniform convergence and the Lipschitz convergence coincide on the set of admissible functions of problem $(\mathrm{P})$ with that additional restriction (see also Remark 4), Theorem 4.4 of [24] is a particular case of Theorem 2 from [26], which was used in the proof of Theorem 3.3 above.

It follows from the results of Morrey's monograph [23] that, if in problem $(\mathrm{P})$ the set of admissible functions is extended to the class of functions from the space $W_{1}^{1}(\Omega)$ whose restriction into $\Gamma$ is $\varphi, E_{1}(\varphi)$, and if integrand $f$ has continuous partial derivatives with respect to $\zeta_{1}, \ldots, \zeta_{n}$, then problem $\left(\mathrm{PR}_{1}\right)$ has a solution. On the other hand, many cases are known, where a solution of problem $\left(\mathrm{PR}_{1}\right)$ (with the class of admissible functions $E_{1}(\varphi)$ ) is Lipschitz, i.e. belongs to the subspace $W_{\infty}^{1}(\Omega) \subset W_{1}^{1}(\Omega)$ (see [23] and [28]). These results, in combination with Theorem 3.3, allow one to formulate different assertions on the existence of minimizing Lipschitz converging sequences of the problem $(\mathrm{P})$.

\section{Existence of Lipschitz Convergent Minimizing Sequences and the Lavrentiev Phenomenon}

We apply in this section Theorem 3.3 to establish the existence of the Lipschitz convergent minimizing sequences and therefore the absence of the Lavrentiev phenomenon in one-dimensional variational problems. We shall need the following two lemmas.

Lemma 4.1 Let $f: \bar{\Omega} \times \mathbb{R}^{m} \times \mathbb{R}^{n} \rightarrow \mathbb{R}$ satisfy the assumptions of Theorem 2.5 and be locally Lipschitz in $(x, v)$ uniformly in $t$, that is, for each bounded subset $C$ of $\mathbb{R}^{m} \times \mathbb{R}^{n}$ there exists a constant $K$ such that, for all $(x, u),\left(x^{\prime}, u^{\prime}\right)$ in $C$ and for all $t \in \bar{\Omega}$,

$$
\left|f(t, x, u)-f\left(t, x^{\prime}, u^{\prime}\right)\right| \leq K\left\|\left(x-x^{\prime}, u-u^{\prime}\right)\right\| .
$$

Then the convexification $f(t, x, u)$ is also locally Lipschitz in $(x, u)$ uniformly in $t$.

Proof First show that $f^{* *}$ is locally Lipschitz in $x$ uniformly in $(t, u) \in \bar{\Omega} \times C_{1}$, where $C_{1}$ is an arbitrary bounded part of $\mathbb{R}^{n}$. Let $R>0$ be such that the ball $B_{R}^{n}$ in $\mathbb{R}^{m}$ with radius $R$ and center at the origin contains $C_{1}$. Then by Theorem 2.5 , there exists $R^{\prime}>R$ such that

$$
f^{* *}(t, x, u)=\min \left\{\sum_{k=1}^{n+1} \alpha_{k} f\left(t, x, u_{k}\right) \mid \alpha_{k} \geq 0, \sum_{k} \alpha_{k}=1, u_{k} \in B_{R^{\prime}}^{n}, \sum_{k} \alpha_{k} u_{k}=u\right\},
$$

for an arbitrary $t \in \bar{\Omega}, x \in B_{R}^{m}$, and $u \in B_{R}^{n}$. Let $K$ be the Lipschitz coefficient in (4.1) for 
compact $C=B_{R+1}^{m} \times B_{R^{\prime}}^{n}$. It easily follows from (4.1) and (4.2) that

$$
\left|f^{* *}(t, x, u)-f^{* *}(t, x, u)\right| \leq K\left\|x-x^{\prime}\right\|_{m},
$$

for each $t \in \Omega, x_{R}^{\prime m}, u \in B_{R}^{n}$.

Now show that $f^{* *}$ is locally Lipschitz in $u$ uniformly in $(t, x) \in \bar{\Omega} \times B_{R}$. Let $u, u_{R}^{\prime n}$. Then

$$
f^{* *}(t, x, u)=\sum_{k} \alpha_{k} f\left(t, x, u_{k}\right),
$$

for $u_{k} \in B_{R^{\prime}}$ and $\alpha_{k} \geq 0, k=1, \ldots, n+1$ as in (4.2). Put $u_{k}^{\prime}=u_{k}+\left(u^{\prime}-u\right), k=1, \ldots, n+1$. Then $\sum_{k} \alpha_{k} u_{k}^{\prime}=u^{\prime}$ and

$$
\sum_{k} \alpha_{k} f\left(t, x, u_{k}^{\prime}\right) \leq \sum_{k} f\left(t, x, u_{k}\right)+K\left\|u_{k}^{\prime}-u\right\|=f^{* *}(t, x, u)+K\left\|u^{\prime}-u\right\|_{n} .
$$

This implies

$$
f^{* *}(t, x, u) \leq f^{* *}(t, x, u)+K\left\|u^{\prime}-u\right\|_{n}
$$

Symmetrically,

$$
f^{* *}(t, x, u) \leq f^{* *}(t, x, u)+K\left\|u^{\prime}-u\right\|_{n} .
$$

Uniting the last two inequalities, we obtain

$$
\left|f^{* *}(t, x, u)-f^{* *}\left(t, x, u^{\prime}\right)\right| \leq K\left\|u^{\prime}-u\right\|_{n} .
$$

Thus, $f^{* *}$ is locally Lipschitz in $x$ locally uniformly in $(t, u)$ and is Lipschitz in $u$ locally uniformly in $(t, x)$. It easily follows from this, that $f^{* *}$ is locally Lipschitz in $(x, u)$ uniformly in $t$.

Lemma 4.2 Let $f:[a, b] \times \mathbb{R}^{m} \times \mathbb{R}^{n} \rightarrow \mathbb{R}$ be a lower semicontinuous function differentiable with respect to $t$ and such that $f(t, x, u) \geq \gamma_{1}(t)$ for all $(t, x, u) \in[a, b] \times \mathbb{R}^{m} \times \mathbb{R}^{n}$, where $\gamma_{1}(\cdot) \in$ $L_{1}[a, b]$, and let $f$ satisfy the growth condition $\frac{f(t, x, u)}{\|u\|} \rightarrow \infty$ for $\|u\| \rightarrow \infty$ locally uniformly in $(t, x)$, and the inequality

$$
\left|f_{t}(t, x, u)\right| \leq c|f(t, x, u)|+\gamma_{2}(t), \quad \forall(t, x, u),
$$

where $\gamma_{2}(\cdot) \in L_{1}[a, b]$ and $c$ is a positive constant. Then there exists $\gamma(\cdot) \in L_{1}[a, b]$ such that

$$
f_{t}^{* *}(t, x, u)|\leq c| f^{* *}(t, x, u) \mid+\gamma(t),
$$

for each $(t, x, u)$ such that function $\sigma \mapsto f^{* *}(\sigma, x, u)$ is differentiable at $t$.

Proof We assume, without loss of generality, that $\gamma_{1}(t) \leq 0$ and $\gamma_{2}(t) \geq 0$. By the assumption $f(t, x, u) \geq \gamma_{1}(t)$ and then

$$
|f(t, x, u)| \leq\left|f(t, x, u)-2 \gamma_{1}(t)\right|, \quad \forall(t, x, u) \in[a, b] \times \mathbb{R}^{m} \times \mathbb{R}^{n} .
$$

Using this in (4.4), we obtain

$$
\left|f_{t}(t, x, u)\right| \leq c\left|f(t, x, u)-2 \gamma_{1}(t)\right|+\gamma_{2}(t), \quad \forall(t, x, u) \in[a, b] \times \mathbb{R}^{m} \times \mathbb{R}^{n} .
$$

Since $f(t, x, u)-2 \gamma_{1}(t) \geq 0$,

$$
-c f(t, x, u)-\gamma(t)<f_{t}(t, x, u)<c f(t, x, u)+\gamma(t), \quad \forall(t, x, u) \in[a, b] \times \mathbb{R}^{m} \times \mathbb{R}^{n},
$$


where $\gamma(t)=\gamma_{2}(t)-2 c \gamma_{1}(t)+1$. Fix $\left(t_{0}, x_{0}\right) \in[a, b] \times \mathbb{R}^{m}$. Then for each $u \in \mathbb{R}^{n}$ there exists $\delta\left(t_{0}, x_{0}, u\right)>0$ such that

$f\left(t_{0}, x_{0}, u\right)-\left[c f\left(t_{0}, x_{0}, u\right)+\gamma\left(t_{0}\right)\right]\left(t-t_{0}\right)<f\left(t, x_{0}, u\right)<f\left(t_{0}, x_{0}, u\right)+\left[c f\left(t_{0}, x_{0}, u\right)+\gamma\left(t_{0}\right)\right]\left(t-t_{0}\right)$, for all $u \in \mathbb{R}^{n}$ and $0<t-t_{0}<\delta\left(t_{0}, x_{0}, u\right)$ or

$$
\begin{aligned}
{\left[1-c\left(t-t_{0}\right)\right] f\left(t_{0}, x_{0}, u\right)-\gamma\left(t_{0}\right)\left(t-t_{0}\right) } & <f\left(t, x_{0}, u\right) \\
& <\left[1+c\left(t-t_{0}\right)\right] f\left(t_{0}, x_{0}, u\right)+\gamma\left(t_{0}\right)\left(t-t_{0}\right),
\end{aligned}
$$

for all $u \in \mathbb{R}^{n}, 0<t-t_{0}<\delta\left(t_{0}, x_{0}, u\right)$.

By Theorem 2.5, there are $\left(u_{k}, \alpha_{k}\right) \in \mathbb{R}^{n} \times \mathbb{R}^{1}(k=1, \ldots, n+1)$ for fixed $u_{0}$ such that

$$
f^{* *}\left(t_{0}, x_{0}, u_{0}\right)=\sum_{k=1}^{n+1} \alpha_{k} f\left(t_{0}, x_{0}, u_{k}\right), \quad \sum_{k=1}^{n+1} \alpha_{k} u_{k}=u_{0}, \alpha>0, \quad \text { and } \sum_{k+1}^{n+1} \alpha_{k}=1 .
$$

Let $\left(t_{0}, x_{0}, u_{0}\right) \in[a, b] \times \mathbb{R}^{m} \times \mathbb{R}^{n}$ be such that function $\sigma \mapsto f^{* *}\left(\sigma, x_{0}, u_{0}\right)$ is differentiable at $t_{0}$. Multiplying by $\alpha_{k}$ the second of inequalities (4.5) for $u=u_{k}$ and summing up, we obtain

$$
\sum_{k=1}^{n+1} \alpha_{k} f\left(t, x_{0}, u_{k}\right) \leq\left[1+c\left(t-t_{0}\right)\right] f^{* *}\left(t_{0}, x_{0}, u_{0}\right)+\gamma\left(t_{0}\right)\left(t-t_{0}\right)
$$

for $0<t-t_{0}<\delta$, where $\delta=\min \left\{\min _{1 \leq k \leq n+1} \delta\left(t_{0}, x_{0}, u_{k}\right), \frac{1}{2 c}\right\}$, and then by the definition of $f^{* *}$, we have

$$
f^{* *}\left(t, x_{0}, u_{0}\right) \leq\left[1+c\left(t-t_{0}\right)\right] f^{* *}\left(t_{0}, x_{0}, u_{0}\right)+\gamma\left(t_{0}\right)\left(t-t_{0}\right) \quad \text { for } 0<t-t_{0}<\delta .
$$

Similarly, for an arbitrary $0<t-t_{0}<\delta$, let

$$
f^{* *}\left(t, x_{0}, u_{0}\right)=\sum_{k=1}^{n+1} \beta_{k}(t) f\left(t, x_{0}, v_{k}(t)\right), \quad \sum_{k=1}^{n+1} \beta_{k}(t) v_{k}(t)=u_{0}, \beta_{k}(t) \geq 0, \quad \sum_{k=1}^{n+1} \beta_{k}(t)=1 .
$$

Multiplying by $\beta_{k}(t)$ the first of inequalities (4.5) for $u=v_{k}(t)$ and summing up, we obtain

$$
\left[1-c\left(t-t_{0}\right)\right] \sum_{k=1}^{n+1} \beta_{k}(t) f\left(t_{0}, x_{0}, v_{k}(t)\right)-\gamma\left(t_{0}\right)\left(t-t_{0}\right) \leq f^{* *}\left(t, x_{0}, u_{0}\right)
$$

and then since $1-c\left(t-t_{0}\right)>0$, by the definition of $f^{* *}$, we have

$$
\left[1-c\left(t-t_{0}\right)\right] f^{* *}\left(t_{0}, x_{0}, u_{0}\right)-\gamma\left(t_{0}\right)\left(t-t_{0}\right) \leq f^{* *}\left(t, x_{0}, u_{0}\right) \quad \text { for } 0<t-t_{0}<\delta .
$$

Using (4.6) and (4.7), we obtain

$$
-c f^{* *}\left(t_{0}, x_{0}, u_{0}\right)-\gamma\left(t_{0}\right) \leq \frac{f^{* *}\left(t, x_{0}, u_{0}\right)-f^{* *}\left(t_{0}, x_{0}, u_{0}\right)}{t-t_{0}} \leq c f^{* *}\left(t_{0}, x_{0}, u_{0}\right)+\gamma\left(t_{0}\right),
$$

for each $t$ such that $0<t-t_{0}<\delta$. Since $f$ is assumed to be differentiable with respect to $t$, passing to limit as $t$ tends to $t_{0}$, we obtain

$$
-c f^{* *}\left(t_{0}, x_{0}, u_{0}\right)-\gamma\left(t_{0}\right) \leq f_{t}^{* *}\left(t_{0}, x_{0}, u_{0}\right) \leq c f^{* *}\left(t_{0}, x_{0}, u_{0}\right)+\gamma\left(t_{0}\right),
$$

or

$$
\left|f_{t}^{* *}\left(t_{0}, x_{0}, u_{0}\right)\right| \leq c\left|f^{* *}\left(t_{0}, x_{0}, u_{0}\right)\right|+\gamma\left(t_{0}\right)
$$

In the following theorems problems $(\mathrm{P})$ and $\left(\mathrm{PR}_{1}\right)$ will be considered in the one-dimensional case and with the set of absolutely continuous functions on $[a, b]$ satisfying the boundary condition in problem $(\mathrm{P})$. 
Theorem 4.3 Let $f:[a, b] \times \mathbb{R} \times \mathbb{R} \rightarrow \mathbb{R}$ be independent from $t$, locally Lipschitz and satisfy the growth condition $f(t, x, u) \geq-\alpha|x|+\varphi(|u|)$ for all $(x, u) \in \mathbb{R} \times \mathbb{R}$, where $\varphi:[0, \infty) \rightarrow \mathbb{R}$ is a function satisfying the condition $\frac{\varphi(r)}{r} \rightarrow \infty$ as $r \rightarrow \infty$. Then there exists a minimizing sequence of problem $(\mathrm{P})$, where $\Gamma=\{a, b\}$ converging Lipschitz to some function (a solution of problem $\left.\left(\mathrm{PR}_{1}\right)\right)$.

Proof By Lemma 4.1, the function $f^{* *}$ is locally Lipschitz. Then it is clear that $f^{* *}$ satisfies all conditions of Theorem 4.3 and therefore, those of Theorem 2.1 from Clarke and Vinter [29]. Hence, problem $\left(\mathrm{PR}_{1}\right)$ has an absolutely continuous solution $x_{0}(\cdot)$. By Corollary 3.1 from Clarke and Vinter $[29] x_{0}(\cdot) \in W_{\infty}^{1}[a, b]$, i.e., $x(\cdot)$ is Lipschitz. By Theorem 3.3, whose conditions are obviously satisfied, there exists a sequence $x_{k}(\cdot)(k \in \mathbb{N})$ converging Lipschitz to $x_{0}(\cdot)$ and such that

$$
\lim _{k} J_{f}\left(x_{k}(\cdot)\right)=J_{f}\left(x_{0}(\cdot)\right) .
$$

Since $J_{f^{* *}}\left(x_{0}(\cdot)\right)=\inf \left(P R_{1}\right)$ and $\inf (P) \geq \inf \left(P R_{1}\right)$, it follows from here that $x_{k}(\cdot)(k \in \mathbb{N})$ is a minimizing sequence of $(\mathrm{P})$.

Theorem 4.4 Let $f(t, x, u):[a, b] \times \mathbb{R} \times \mathbb{R} \rightarrow \mathbb{R}$ be a locally Lipschitz function differentiable with respect to $t$, and such that $f(t, x, u) \geq \gamma_{1}(t)$ for all $(t, x, u) \in[a, b] \times \mathbb{R} \times \mathbb{R}$, where $\gamma_{1}(\cdot) \in$ $L_{1}[a, b]$, and let $f$ satisfy the growth condition $f(t, x, u) \geq-\alpha|x|+\varphi(|u|)$ for all $(t, x, u)$ in $[a, b] \times$ $\mathbb{R} \times \mathbb{R}$, where $\frac{\varphi(r)}{r} \rightarrow \infty$ as $r \rightarrow \infty$, and let for an arbitrary bounded set $S \subset \mathbb{R}$ there exist a constant $c$ and a summable function $\gamma(\cdot)$ such that

$$
\left|f_{t}(t, x, u)\right| \leq c|f(t, x, u)|+\gamma(t)
$$

for all points $(t, x, u)$ in $[a, b] \times S \times \mathbb{R}$ at which the mapping $(\sigma, y) \mapsto f(\sigma, y, u)$ is differentiable. Then there exists a minimizing sequence of problem $(\mathrm{P})$ (with $\Gamma=\{a, b\})$ Lipschitz converging to some function (a solution of problem $\left(\mathrm{PR}_{1}\right)$ ).

Proof Proof is similar to the proof of Theorem 4.3: Corollary 3.2 from [24] and Lemma 4.2 play roles parallel to those of Corollary 3.1 from [24] and Lemma 4.1 in the proof of Theorem 4.3.

Corollary 4.5 Under the assumptions of Theorem 4.3 or Theorem 4.4 in one-dimensional variational problem $(\mathrm{P})$, the Lavrentiev phenomenon does not occur.

\section{References}

[1] Lavrentiev, M. M.: Sur quelques problemes du calcul des variations. Annali di Matematica, Pura et Applic., 4, 7-28 (1926)

[2] Cesari, L.: Optimization-Theory and Applications, Springer Verlag, New York-Heidelberg-Berlin, 1983

[3] Tihomirov, V. M.: A remark on Lavrentiev phenomenon. Turkish J. Math., 18, 111-113 (1994)

[4] Ball, J. M., Mizel, V. J.: Singular minimizers for regular one-dimensional problems in the calculus of variations. Bull. Amer. Math. Soc., 11, 143-146 (1984)

[5] Loewen, Ph. D.: On the Lavrentiev phenomenon. Canad. Math. Bull., 30, 102-108 (1987)

[6] Buttazzo, G., Mizel, V.: Interpretation of the Lavrentiev pnenomenon by relaxation. J. Funct. Anal., 110, 434-460 (1992)

[7] Buttazzo, G., Belloni, M.: A Survey of Old and Recent Results about the Gap Phenomenon. In: R. Lucchetti, J. Revalski (Eds.), Recent Developements in Well-Posed Variational Problems, Math. Appl. Vol. 331, Kluwer Academic Publishers, Dodrecht, 1995 
[8] Mizel, V. J.: New developments concerning the Lavrentiev phenomenon. In: Calculus of Variations and Differential Equations (Haifa, 1998), Chapman \& Hall/CRS Res. Notes Math. Vol. 410, Roca Raton, FL, 2000

[9] Ambrosio, L., Ascenzi, O., Buttazzo, G.: Lipscitz regularity for minimizers of integral functionals with highly discontinuous integrands. J. Math. Anal. Appl., 142, 301-316 (1989)

[10] De Arcangelis, R.: The Lavrentiev phenomenon for quadratic functionals. Proc. Roy. Soc. Edinburgh Sect. A, 125, 329-339 (1995)

[11] Buttazzo, G., Mizel, V.: On a gap phenomenon for isoperimetrically constrained variational problems. $J$. Convex Anal., 2, 87-101 (1995)

[12] Ferriero, A.: The Lavrentiev Phenomenon in the Calculus of Variations, Thesis, Universita degli Studi de Milano-Bicocca, 2004

[13] Sychev, M. A.: On the question of regularity of the solutions of variational problems. Sb. Math., 75 , 535-556 (1993)

[14] Ball, J. M., Knowles, G.: A numerical method for detecting singular minimizers. Numer. Math., 51, 181197 (1987)

[15] Clarke, F. H.: Methods of Dynamic and Nonsmooth Optimization, Capital City Press, Montpeller, 1989

[16] Ekeland, I., Temam, R.: Convex Analysis and Variational Problems, North Holland, Amsterdam, 1976

[17] Klee, V. L.: Convex sets in linear spaces. Duke Math. J., 18, 443-466 (1951)

[18] Hüsseinov, F. V.: A note on the closedness of the convex hull and its applications. J. Convex Anal., 6, 387-394 (1999)

[19] Hiriart-Urruty, J. B., Lemarechal, C.: Convex Analysis and Minimization Algorithms, Vol. 2, Springer, Berlin-New York, 1993

[20] Griewank, A., Rabier, P. J.: On the smoothness of convex envelopes. Trans. Amer. Math. Soc., 322, 691-709 (1990)

[21] Cellina, A.: The classical problem of the calculus of variations in the autonomous case: Relaxation and Lipschitzianity of Solutions. Trans. Amer. Math. Soc., 356, 415-426 (2004)

[22] Rockafellar, R. T.: Convex Analysis, Princeton University Press, Princeton, 1970

[23] Morrey, C. B.: Multiple Integrals in the Calculus of Variations, Grundlehren Math. Wiss., Bd. 139, Springer, Berlin-Heidelberg-New York, 1966

[24] Marcellini, P., Sbordone, C.: Semicontinuity problems in the calculus of variations. Nonlinear Anal., 4, 241-257 (1980)

[25] Dunford, N., Schwartz, J. T.: Linear Operators, Part I: General Theory, Interscience, New York, 1958

[26] Guseinov, F. V.: On the question of extension of multidimensional variational problems. Izv. Math., 28, 1-19 (1987)

[27] Serrin, J.: On the definition and properties of certain variational integrals. Trans. Amer. Math. Soc., 101, 139-167 (1961)

[28] Ladyzhenskaya, O., Uraltseva, N. N.: Linear and Quasilinear Differential Equations of Elliptic Type, Nauka, Moscow, 1964

[29] Clarke, F. H., Vinter, R. B.: Regularity properties of solutions to the basic problem in the calculus of variations. Trans. Amer. Math. Soc., 289, 73-79 (1985) 